\title{
Einfluß der Gewichtsreduktion auf Metabolite des Kohlenhydrat- und Fettstoffwechsels und auf das Verhalten des Seruminsulins bei Adipositas*
}

\author{
H. Liebermeister, H. Daweke, F.A. Gries, W.H. Schilding, D. Grüneklee, G. Probst, und K. Jahnket.
}

2. Medizinische Universitätsklinik und Diabetes-Forschungsinstitut, Universität Düsseldorf

(Direktor: Prof. Dr. K. OBERDTSSE)

Eingegangen am 20. Juni 1967

\begin{abstract}
Influence of weight reduction on carbohydrate and fat metabolism and on serum insulin response in obesity

Summary. To find out which of the metabolic changes observed in obesity are reversible by a reducing diet, we examined 37 obese out-patients with a mean overweight of $71 \%$ BRocA. A manifest diabetes mellitus and endocrine diseases were excluded by clinical means. $20 \mathrm{sub}$ jects showed disturbances of carbohydrate-tolerance. Oral and i.v. glucose-tolerance tests gave normal results in 17. Both subgroups were put on a 1000 cal. mixed diet and had mean weight-losses of 21.2 , vs. $17.5 \mathrm{~kg}$, corresponding to $34 \%$ vs. $28 \%$ BRocA, i.e. less than half of their overweight. - The following changes were observed in the subgroup with impaired carbohydrate-tolerance: 1. Significant improvement of glucose-tolerance with beginning normalization of fasting blood sugar, $120^{\prime}$ value after oral glucose and of the $k$-values for i.v. and oral glucose-tolerance tests. - 2. Highly significant reduction of the elevated fasting values for free serum glycerol with normalization of the quotient: free fatty acids/glycerol in fasting serum. - 3. Significant fall of the high levels for insulin-like activity and for immunologically reacting insulin. - Under identical conditions the subgroup with normal carbohydrate-tolerance showed only a moderate increase of free fatty acids $90^{\prime}$ and $120^{\prime}$ after glucose. In the obese group as a whole, we found a reduction of serum esterified fatty acids under low calorie diet. - The changes described under $1-3$ were more pronounced with this dietary treatment than in fasting periods generally described until now, and occurred predominantly in the subgroup with impaired carbohydrate-tolerance. Our findings indicate that hyperinsulinism held responsible for obesity up to now by some authors is probably adap. tive.
\end{abstract}

Influence d'une réduction de poids sur le métabolisme des carbohydrates et des lipides et sur la réponse de l'insuline sérique dans l'obésité

Résumé. Pour voir jusqu'à quel degré les déviations métaboliques qu' on observe dans l'obésité sont réversibles par un régime de réduction, nous avons examiné 37 personnes obèses avec un excès de poids moyen de $71 \%$ d'après BRoCA. D'abord nous avons exclu la présence d'un diabète sucré ot d'une maladie endocrinienne. 20 personnes montraient des anomalies de la glycorégulation tandis que chez 17 les tests de l'hyperglycémie provoquée par voie intraveineuse et orale donnaient des résultats normaux. Un régime de 1000 cal. par jour a été institué pour les deux groupes et nous avons enregistré une perte de poids moyenne de $21.2 \mathrm{~kg}$ pour la première et de $17.5 \mathrm{~kg}$ pour la seconde, soit de 34 ou de $28 \%$ d'après Broca,

* Mit dankenswerter Unterstützung der Deutschen Forschungsgemeinschaft und des Landesamtes für Forschung des Landes Nordrhein-Westfalen. - Die Befunde wurden auszugsweise auf dem 6 . Kongreß der Internationalen Diabetes Föderation vom 30. 7. - 4. 8. 1967 in Stockholm vorgetragen. c'est à dire moins que la moitié de l'excès de poids. - Le groupe avec glycorégulation anormale montrait les changements suivants: - 1. Amélioration significative de la tolérance glucidique avec normalisation partielle du taux de glucose sanguin à jeun et 120 min après glucose par voie orale, ainsi que des coefficients $k$ pour les tests d'hyperglycémie provoquée par voie intraveineuse et orale. -2 . Une chute prononcée et significative des taux très élevés du glycérol libre dans le sang à jeun avec normalisation du quotient: acides gras libres/glycérol à jeun. - 3. Réduction significative des taux élevés de l'activité insulinosemblable et de l'insuline immunoréactive dans le sang. - Dans les mêmes conditions nous avons trouvé dans le groupe avec glycorégulation intacte seulement une augmentation légère des acides gras libres sanguins 90 et $120 \mathrm{~min}$ après glucose. Le groupe total des obèses montrait une réduction significative des acides gras estérifiés dans le sang à jeun. - Les résultats caractérisés sous $1-3$ sont plus prononcés avec ce régime de réduction qu'après des cures de carence alimentaire totale et se trouvent surtout dans le groupe avec glycorégulation anormale. Ils indiquent que l'hyperinsulinisme accusé jusqu'ici comme pathogénique pour l'obésité est plutôt de nature adaptive.

Zusammenfassung. Um Anhaltspunkte dafür zu gewinnen, welche der bei der Fettsucht beobachteten Stoffwechselanomalien durch eine Reduktionsdiät rückbildungsfähig sind, untersuchten wir unter ambulanten Bedingungen 37 adipöse Patienten mit einem mittleren U̇bergewicht von $71 \%$ nach Broca. Ein klinisch-manifester Diabetes und endokrine Erkrankungen wurden ausgeschlossen. 20 der Probanden zeigten Störungen der Kohlenhydrattoleranz. Bei 17 lagen der i.v. und der orale Glucosetoleranz-Test im Normbereich. Beide Teilkollektive behandelten wir mit einer 1000 Cal.-Mischkost und sahen darunter eine Gewichtsabnahme von 21.2 bzw. 17.5 $\mathrm{kg}$, entsprechend $34 \mathrm{bzw}$. $28 \%$ nach BROCA, d.h. im Durchschnitt etwas weniger als die Hälfte des bestehenden U̇bergewichtes. - Beim Teilkollektiv mit gestörter Kohlenhydrattoleranz traten dabei folgende Veränderungen ein:- - 1. Signifikante Besserung der Glucosetoleranz mit weitgehender Normalisierung des Nüchternblutzuckers und des 120 Minuten-Wertes nach oraler Glucosegabe, sowie der $k$-Werte für die i.v. und oralen Glucosetoleranz-Teste. - 2. Ein hochsignifikanter Abfall der stark erhöhten Nüchternwerte für das freie Serumglycerin mit Normalisierung des Quotienten: Freie Fettsäuren/ Glycerin im Nüchternblut. - 3. Signifikanter Rückgang der überhöhten Werte für die insulinähnliche Alktivität und für das immunologisch reagierende Insulin. - Unter den gleichen Bedingungen fanden wir im Teilkollektiv mit normaler Kohlenhydrattoleranz lediglich einen mäßigen Anstieg der freien Fettsäuren $90^{\prime}$ und $120^{\prime}$ nach Glucose. - Im Gesamtkollektiv der Fettsüchtigen war unter der Reduktionsdiät ein signifikanter Rückgang der Esterfettsäuren festzustellen. - Die unter $1-3$ geschilderten Veränderungen traten unter dieser Diätbehandlung deutlicher hervor als bei den bisher beschriebenen Fastenkuren und betreffen vorwiegend das Teilkollektiv mit gestörter 
Kohlenhydrattoleranz. Die Befunde deuten daraufhin, daß der Hyperinsulinismus, der von einigen Autoren als ursächlich für die Fettsucht angesehen wird, eher adaptiver Natur ist.
Key-words: Weight reduction, low-calorie-diet, FFA, esterified fatty acids, glycerol, glucose tolerance, immunoreactive Insulin (IRI), insulinlike activity as measured on adipose tissue (FILA), and on muscle tissue (MILA).
Über die Veränderungen des Kohlenhydrat- und Fettstoffwechsels bei Adipositas mit und ohne manifesten Diabetes existieren zahlreiche Untersuchungen $[2,3,6,8,9,10,16,17,24,27,32,33,35,45,55,56$, $61,65,74,76,79,92]$. Auch über die Rückbildung einiger dieser Veränderungen bei diätetisch bedingter Gewichtsreduktion wurde mehrfach berichtet $[6,7,12$, $29,31,32,38,39,44,45,58,59,74,76,77,78,83,87]$. Die Gewichtsabnahme erfolgte dabei jedoch im allgemeinen durch völligen Nahrungsentzug über verschieden lange Zeiträume, so daß sich eine Hungerketose ausbildete $[7,31,38,39,76,77,78,83]$. Ziel unserer Untersuchungen war es, die Paardifferenzen der Änderung verschiedener Parameter des Fett- und Kohlehydratstoffwechsels zu prüfen, die bei ausgeprägter Gewichtsreduktion infolge langfristiger unterkalorischer Ernährung bei Vermeidung von stärker ausgeprägter Hungerketose auftritt.

\section{Patientengut und Untersuchungsmethoden}

Das Beobachtungskollektiv umfaßte 37, vorwiegend weibliche Personen mit stabiler Fettsucht aus unserer Stoffwechselambulanz. Die klinischen Daten sind in Tabelle 1 zusammengetragen. Alle Untersuchungen erfolgten unter ambulanten Bedingungen.

\section{Table 1. Patients}

\begin{tabular}{llll}
\hline & \multicolumn{2}{l}{ Glucose tolerance } & \\
\cline { 2 - 3 } & normal & impaired & total \\
\hline Number & 17 & 20 & 37 \\
Females & 16 & 13 & 29 \\
Age (years) & $37(14-70)^{\mathrm{a}} 45(21-62)$ & $41(14-70)$ \\
Broca-Index (\%) & $173 \pm 7 \mathrm{~b}$ & $169 \pm 5$ & $171 \pm 4$ \\
Weight-loss in & $\mathbf{2 8 \pm 2}$ & $\mathbf{3 4 \pm 3}$ & $\mathbf{3 1 \pm 2}$ \\
Broca \% & & & \\
Starting-weight & $101.1 \pm 3.9$ & $103.4 \pm 4.4$ & $102.2 \pm 3.6$ \\
(kg) & & & \\
Weight-loss (kg) & $17.5 \pm 1.2$ & $21.2 \pm 1.8$ & $19.5 \pm 1.2$ \\
\hline
\end{tabular}

a range in brackets
b mean $\bar{x} \pm \mathrm{SEM}$

Bei allen Probanden wurde durch eine eingehende klinische Untersuchung das Vorliegen einer endokrinen Erkrankung sowie eines klinischen Diabetes ausgeschlossen.

Während einer Vorperiode von 1-3 Wochen, im Durchschnitt 13 Tage, wurde das Gewichtsverhalten bei frei gewählter, nicht eingeschränkter Ernährungsaufnahme überprüft. Dabei ergab sich eine unbedeutende Gewichtsabnahme von $0.39 \pm 0.11 \mathrm{~kg}$. Nach Abschluß der Vorperiode wurden folgende Untersu- chungen durchgeführt: 1. Oraler Standard $(100 \mathrm{~g})$ Glucosetoleranztest (o.GTT) [79]. 2. Intravenöser (25 g) Glucosetoleranztest (i.v. GTT) [79] mit Bestimmung des Glucose-Assimilations-Koeffizienten nach ConARD [13]. 3. Freie Fettsäuren (FFS) $[19$, 52]. 4. Freies Glycerin [49]. 5. Esterfettsäuren (EFS) [73]. 6. Triglyceride [50]. 7. Cholesterin [84]. 8. Azetoazetat (AzAz) [89]. 9. Beta-Hydroxybutyrat (HBS) [89]. 10. Insulinähnliche Aktivität am Fettgewebe (FILA) [54]. 11. Immunologisch reagierendes Insulin (IRI) [36]. 12. Insulinähnliche Aktivität am Muskel (MILA) [51, $69]$.

Die Normalwerte, die wir bei diesen Untersuchungen erhalten, sind in Tabelle 2 zusammengefaßt.

Eine normale Glucosetoleranz wurde nur dann angenommen, wenn der Assimilations-Koeffizient $K \times 10^{2}$ (13) des i.v. GTT größer als 1.40 und der planimetrisch ermittelte $K$-Wert ${ }^{1}$ (72) des o. GTT kleiner als 2.40 ausfielen, d.h. wenn beide im Streubereich $(\bar{x} \pm 2$ SD) der jeweiligen Norm lagen.

Der Vorperiode und den Voruntersuchungen schloß sich die Reduktionsperiode an: Zunächst erfolgte eine eingehende Belehrung über die Zubereitung einer 1000 Kalorien Mischkost unter besonderer Berücksichtigung des Eiweiß-, Vitamin- und Ballaststoffgehaltes durch unsere Ernährungsberaterinnen. Wir wiesen die Probanden an, die täglich zugeführte Kalorien- und Eiweißmenge anhand der kleinen Nährwerttabelle der Deutschen Gesellschaft für Ernährung (50) zu berechnen und in Form eines Ernährungsprotokolls zu notieren. Sie stellten sich in 2-4-wöchigen Abständen zur Kontrolle des Körpergewichts, ihres Allgemeinbefindens und ihrer Aufzeichnungen vor.

Sobald ein Gewichtsverlust von mindestens $12 \mathrm{~kg}$ eingetreten war, wurden die anfangs durchgeführten Untersuchungen, soweit möglich in vollem Umfange, bei einigen Probanden mehrfach, wiederholt. Die Reduktionsdiät wurde bis zum Tage der Blutentnahme beibehalten. Wo mehrere Untersuchungen am gleichen Patienten vorliegen, haben wir unseren Berechnungen nur die Befunde nach der jeweils größten Gewichtsab. nahme zugrunde gelegt. Die Resultate des Vergleichs der Paardifferenzen vor und unter Reduktionskost sind als Mittelwerte $\bar{x} \pm$ Standardabweichung des Mittelwertes (SEM), die Anzahl der durchgeführten Bestimmungen in Klammern wiedergegeben. Die statistischen Berechnungen erfolgten mit Hilfe des Student- und des Wilcoxon-Tests.

1 Index für die durch Ab̆szisse + Blutzuckerkurve zwischen 0 und 180 min. gebildete Fläche. 


\section{Ergebnisse \\ A. Untersuchungskollektiv:}

Wie aus Tabelle 1 hervorgeht, handelt es sich in der Mehrzahl um jüngere Frauen mit einem Übergewicht von mindestens $+30 \%$ nach Broca, im Durchschnitt $+71 \pm 4 \%$. Dabei stimmen in den beiden Untergruppen mit normaler bzw. gestörter Kohlenhydrattoleranz das Ausmaß des Übergewichtes und die Ge-

\section{B. Einflüsse auf den Kohlenhydratstoffwechsel:}

Glucosetoleranz. Die Reduktionsdiät führte, wie aus Tabelle 3 hervorgeht, in der Gruppe mit normaler Kohlenhydrattoleranz im Durchschnitt zu keiner wesentlichen Veränderung des Blutzuckerverhaltens unter Glucosebelastung. Allerdings fiel bei 2 dieser 17 Patienten nach der Diätperiode der orale GTT pathologisch aus.

Table 2. Confidence intervals $(\bar{x} \pm 2 S E M)$ of normal values

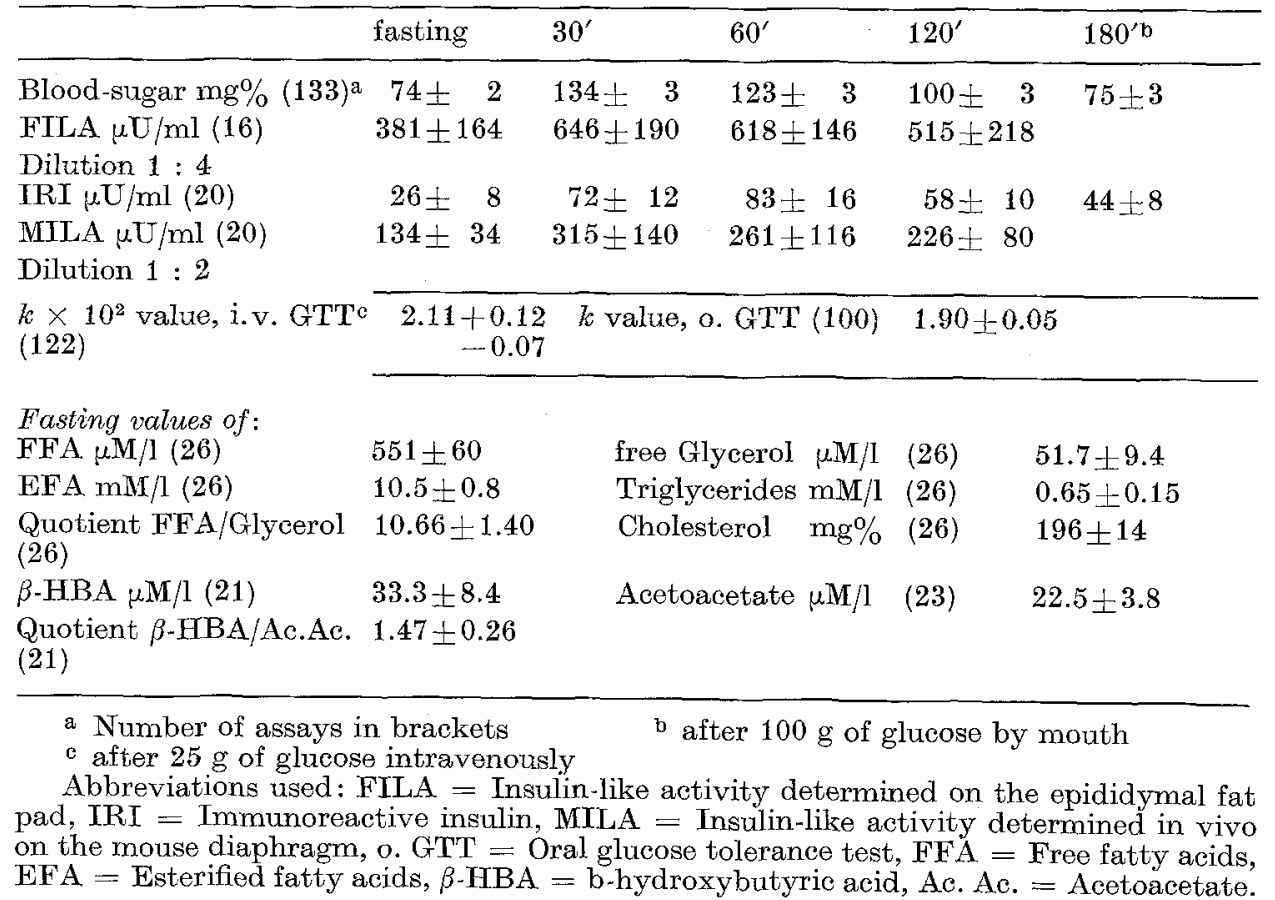

Table 3. Carbohydrate Metabolism before and after weight reduction ${ }^{\mathrm{a}}$

\begin{tabular}{|c|c|c|c|c|c|c|}
\hline & \multicolumn{4}{|c|}{ Glucose Tolerance } & \multirow{2}{*}{\multicolumn{2}{|c|}{ total }} \\
\hline & \multicolumn{2}{|l|}{ normal } & \multicolumn{2}{|l|}{ impaired } & & \\
\hline & before & after & before & after & before & after \\
\hline $\begin{array}{l}\text { fasting blood-sugar } \\
(\mathrm{mg} \%)\end{array}$ & $\begin{array}{l}79 \pm 3 \\
(17)\end{array}$ & $\begin{array}{l}77 \pm 2 \\
(17)\end{array}$ & $\begin{array}{l}86 \pm 3 \\
(20) x x\end{array}$ & $\begin{array}{l}77 \pm 2 \\
(20)\end{array}$ & $\begin{array}{l}83+2 \\
(37) x x\end{array}$ & $\frac{77 \pm 1}{(37)}$ \\
\hline $\begin{array}{l}120^{\prime} \mathrm{b} \text { blood-sugar } \\
(\mathrm{mg} \%)\end{array}$ & $\begin{array}{l}112 \pm 5 \\
(17)\end{array}$ & $\begin{array}{l}114 \pm 5 \\
(17)\end{array}$ & $\begin{array}{l}152 \pm 7 \\
(20) x x x\end{array}$ & $\begin{array}{l}128 \pm 7 \\
(20)\end{array}$ & $\begin{array}{l}134 \pm 4 \\
(37) x\end{array}$ & $\frac{121}{(37)} \pm 4$ \\
\hline $\begin{array}{l}\text { i. } \times 10^{2} \text { value } \\
\text { i. v. } \mathrm{GTT}^{\mathrm{c}}\end{array}$ & $\begin{array}{l}2.04 \pm 0.17 \\
(10)\end{array}$ & $\begin{array}{l}2.01 \pm 0.14 \\
(10)\end{array}$ & $\underset{(18) x x}{1.06 \pm 0.09}$ & $\begin{array}{l}1.52 \pm 0.15 \\
(18)\end{array}$ & $\begin{array}{l}1.40 \pm 0.08 \\
(28) x\end{array}$ & $\begin{array}{l}1.68 \pm 0.11 \\
(28)\end{array}$ \\
\hline $\begin{array}{l}\text { k-value } \\
\text { o. GTT }\end{array}$ & $\begin{array}{l}2.07 \pm 0.05 \\
(17)\end{array}$ & $\begin{array}{l}2.11 \pm 0.07 \\
(17)\end{array}$ & $\begin{array}{l}2.64 \pm 0.10 \\
(20) x x x\end{array}$ & $\frac{2.17}{(20)} \pm 0.12$ & $\begin{array}{l}2.38 \pm 0.06 \\
(37) x\end{array}$ & $\frac{2.14}{(37)} \pm 0.07$ \\
\hline
\end{tabular}

a Mean $\bar{x} \pm$ SEM, number of assays in brackets

$c$ after $25 \mathrm{~g}$ of glucose intravenously

$\mathrm{b}$ after $100 \mathrm{~g}$ of glucose by mouth

$p$ below : $\quad 0.05=x, \quad 0.01=x x, \quad 0.005=x x x, \quad 0.0005=x x x x$

wichtsabnahme gut überein. In dem Teilkollektiv mit normaler Kohlenhydrattoleranz fallen ein höherer Prozentsatz an Frauen und das niedrigere Durchschnittsalter auf. Tabelle 1 zeigt ferner, daß die Probanden im Durchschnitt ihr Übergewicht nur um etwa die Hälfte reduzierten.
Bei den Adipösen mit abnormer Glucosetoleranz traten signifikante Besserungen aller charakteristisehen Werte, wie Nüchternblutzucker, 120-Minuten-Wert nach oraler Belastung und der $K$-Werte für die i.v. und orale GTT ein (Tabelle 3, Abb. 1). Die arithmetischen Mittel aller dieser Parameter lagen während der Re- 
duktionsperiode im Normbereich. Dementsprechend hatte sich bei 10 der 20 Patienten aus der Untergruppe mit abnormer Glucosetoleranz bei der Kontrolluntersuchung das Blutzuckerverhalten normalisiert.

Im Gesamtkollektiv ging der Anteil der Patienten mit abnormer Kohlenhydrattoleranz von 54 auf $32 \%$ zurück ( $p$ unter 0.01).

\section{Einflüsse auf den Fettstoffwechsel:}

a) Freie Fettsäuren und freies Glycerin nüchtern und freie Feltsäuren unter o. GTT. Tabelle 4 und Abb. 1 zeigen den typischen Abfall der freien Fettsäuren nach oraler Glucosegabe. Die Spiegel liegen dabei, vor allem bei den Adipösen mit abnormer KH-Toleranz, wie mehrfach beschrieben, wesentlich über den Normalwerten. Unter der Reduktionsbehandlung erreichen die FFS im allgemeinen etwas höhere Werte; dieser Unterschied ist jedoch nur signifikant beim 90- und 120-Minuten-Wert des Teilkollektivs mit normaler KH-Toleranz.
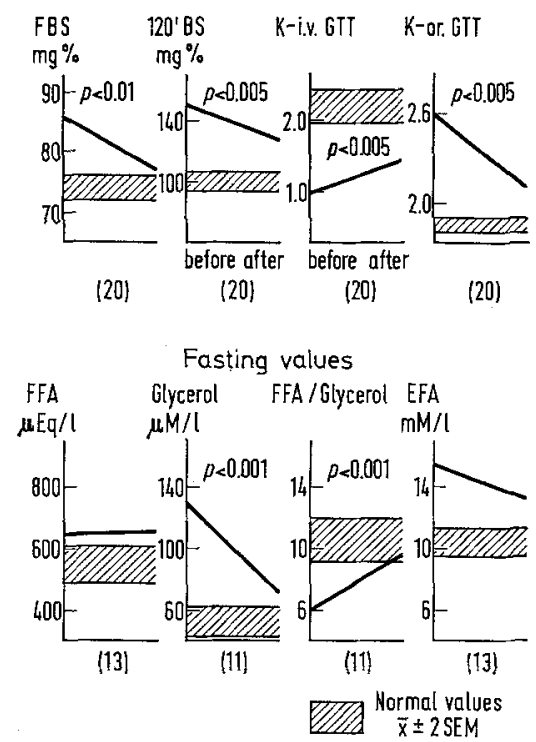

Fig. 1

Table 4. Serum free fatty acids $(\mu E q / l)$ during oral $G T T$ (100 g of glucose) before and after weight reduction

\begin{tabular}{|c|c|c|c|c|c|c|}
\hline & \multicolumn{2}{|c|}{ normal glucose tolerance } & \multicolumn{2}{|c|}{ impaired glucose tolerance } & \multicolumn{2}{|l|}{ total } \\
\hline & $\overline{\text { before }}$ & after & before & after & before & after \\
\hline fasting & $\begin{array}{l}632 \pm 22 \\
(13)\end{array}$ & $\begin{array}{l}669 \pm 40 \\
(13)\end{array}$ & $\begin{array}{l}643 \pm 36 \\
(15)\end{array}$ & $\begin{array}{l}660 \pm 28 \\
(15)\end{array}$ & $\begin{array}{l}638 \pm 22 \\
(28)\end{array}$ & $\begin{array}{l}664 \pm 23 \\
(28)\end{array}$ \\
\hline $30^{\prime}$ & $\begin{array}{l}521 \pm 28 \\
(11)\end{array}$ & $\begin{array}{l}572 \pm 25 \\
\text { (11) }\end{array}$ & $\begin{array}{l}613 \pm 41 \\
(8)\end{array}$ & $\begin{array}{l}553 \pm 50 \\
(8)\end{array}$ & $\begin{array}{l}560 \pm 25 \\
(19)\end{array}$ & $\begin{array}{l}564 \pm 27 \\
(19)\end{array}$ \\
\hline $60^{\prime}$ & $\begin{array}{l}398 \pm 28 \\
(11)\end{array}$ & $\begin{array}{l}460 \pm 21 \\
(11)\end{array}$ & $\begin{array}{l}454 \pm 32 \\
(9)\end{array}$ & $\begin{array}{l}449 \pm 32 \\
(9)\end{array}$ & $\begin{array}{l}423 \pm 21 \\
(20)\end{array}$ & $\begin{array}{l}455 \pm 18 \\
(20)\end{array}$ \\
\hline $90^{\prime}$ & $\begin{array}{l}350 \pm 23 \\
(10) x x\end{array}$ & $\begin{array}{l}424 \pm 18 \\
(10)\end{array}$ & $\begin{array}{l}387 \pm 34 \\
(9)\end{array}$ & $\begin{array}{l}404 \pm 26 \\
(9)\end{array}$ & $\begin{array}{l}367 \pm 20 \\
(19)\end{array}$ & $\begin{array}{l}414 \pm 15 \\
(19)\end{array}$ \\
\hline $120^{\prime}$ & $\begin{array}{l}316 \pm 29 \\
(9) x\end{array}$ & $\begin{array}{l}402 \pm 23 \\
(9)\end{array}$ & $\begin{array}{l}360 \pm 45 \\
(9)\end{array}$ & $\begin{array}{l}373 \pm 41 \\
(9)\end{array}$ & $\begin{array}{l}338 \pm 26 \\
(18)\end{array}$ & $\begin{array}{l}388 \pm 23 \\
(18)\end{array}$ \\
\hline $180^{\prime}$ & $\begin{array}{l}337 \pm 33 \\
(9)\end{array}$ & $\begin{array}{l}428 \pm 33 \\
(9)\end{array}$ & $\begin{array}{l}318 \pm 49 \\
(6)\end{array}$ & $\begin{array}{l}305 \pm 33 \\
(6)\end{array}$ & $\begin{array}{l}329 \pm 31 \\
(15)\end{array}$ & $\begin{array}{l}379 \pm 26 \\
(15)\end{array}$ \\
\hline Fasting & $\frac{95+8}{(10)}$ & $\begin{array}{l}83 \pm 10 \\
(10)\end{array}$ & $\begin{array}{l}129 \pm 13 \\
(11) x x x\end{array}$ & $\begin{array}{l}73 \pm 7 \\
(11)\end{array}$ & $\begin{array}{l}112 \pm 7 \\
(21) x x x x\end{array}$ & $\begin{array}{l}78 \pm 6 \\
(21)\end{array}$ \\
\hline Quotien & $\begin{array}{l}\text { lglycerol }(f \\
6.93 \pm 0.4 \\
(10)\end{array}$ & $8.55 \pm 1.07$ & $\begin{array}{l}5.25 \pm 0.46 \\
\text { (11)xxx }\end{array}$ & $\begin{array}{l}9.62 \pm 0.84 \\
(11)\end{array}$ & $\begin{array}{l}6.05 \pm 0.32 \\
(21) x x x\end{array}$ & $\begin{array}{l}9.11 \pm 0.66 \\
(21)\end{array}$ \\
\hline
\end{tabular}

Number of assays in brackets, mean $\vec{x} \pm$ SEM, for degree of significance see Table 3 .

Table 5. Lipids in fasting serum before and after weight reduction

\begin{tabular}{|c|c|c|c|c|c|c|}
\hline & \multicolumn{2}{|c|}{ Normal glucose tolerance } & \multicolumn{2}{|c|}{ Impaired glucose tolerance } & \multicolumn{2}{|l|}{ Total } \\
\hline & before & after & before & after & before & after \\
\hline Cholesterol $m g \%$ & $\begin{array}{l}231 \pm 11 \\
(14)\end{array}$ & $\begin{array}{l}210 \pm 8 \\
(14)\end{array}$ & $\begin{array}{l}240 \pm 14 \\
(14)\end{array}$ & $\begin{array}{l}220 \pm 12 \\
(14)\end{array}$ & $\begin{array}{l}236 \pm 9 \\
(28)\end{array}$ & $\begin{array}{l}215 \pm 7 \\
(28)\end{array}$ \\
\hline Triglycerides $m M / l$ & $\begin{array}{l}1.02 \pm 0.10 \\
(12)\end{array}$ & $\begin{array}{l}0.80 \pm 0.13 \\
(12)\end{array}$ & $\begin{array}{l}1.33 \pm 0.23 \\
(10)\end{array}$ & $\begin{array}{l}1.10 \pm 0.26 \\
(10)\end{array}$ & $\begin{array}{l}1.15 \pm 0.11 \\
(22)\end{array}$ & $\begin{array}{l}0.94 \pm 0.13 \\
(22)\end{array}$ \\
\hline Esterified Fatty Acid & $\begin{array}{l}M / l \\
14.3 \pm 0.7 \\
(13) x x\end{array}$ & $\begin{array}{l}11.4 \pm 0.6 \\
(13)\end{array}$ & $\begin{array}{l}15.4 \pm 0.9 \\
(15)\end{array}$ & $\begin{array}{l}13.3 \pm 0.7 \\
(15)\end{array}$ & $\begin{array}{l}14.9 \pm 0.6 \\
(28) x x x\end{array}$ & $\begin{array}{l}12.4 \pm 0.5 \\
\{28)\end{array}$ \\
\hline
\end{tabular}


Im Verlauf von Fastenperioden kommt es bekanntlich zu erheblichen Anstiegen der FFS-Spiegel. Demgegenüber beobachteten wir unter der Reduktionsdiät eine wesentlich geringere Erhöhung.

Besonders auffällig erscheint der hoch signifikante Abfall des freien Glycerins, vor allem bei dem Teilkollektiv mit eingeschränkter KH-Toleranz unter der der EFS-Werte im Gesamtkollektiv mit $p$ unter 0.05 eindeutig.

c) Ketokörper im Nüchternserum. Über das Verhalten des Azetoazetat und der Beta-Hydroxybuttersäure lagen nur bei 6 Personen paarweise vergleichbare Werte vor (Tabelle 6). Bei erheblicher Streuung ergab sich ein nicht signifikanter Anstieg der Werte.

Table 6. Ketone bodies in fasting serum before and after weight reduction

\begin{tabular}{|c|c|c|c|c|c|}
\hline \multicolumn{2}{|c|}{ Acetoacetate $\mu \mathrm{M} / \mathrm{l}$} & \multicolumn{2}{|c|}{$\beta$-Hydroxybutyric Acid $\mu \mathrm{M} / \mathrm{l}$} & \multicolumn{2}{|c|}{ Quotient $\frac{\beta \text {-OH Butyric Acid }}{\text { Acetoacetate }}$} \\
\hline before & after & before & after & before & after \\
\hline $\begin{array}{l}40 \pm 11 \\
(6)\end{array}$ & $\begin{array}{l}45 \pm 20 \\
(6)\end{array}$ & $\begin{array}{l}46 \pm 15 \\
(6)\end{array}$ & $\begin{array}{l}86 \pm 49 \\
(6)\end{array}$ & $\begin{array}{l}1.20 \pm 0.25 \\
(6)\end{array}$ & $\begin{array}{l}1.53 \pm 0.18 \\
(6)\end{array}$ \\
\hline
\end{tabular}

Mean $\bar{x} \pm$ SEM, number of assays in brackets, for degree of significance see Table 3 .

Table 7. Seruminsulin after oral GTT $(100 \mathrm{~g})$

\begin{tabular}{|c|c|c|c|c|c|c|}
\hline & \multicolumn{2}{|c|}{ normal GTT } & \multicolumn{2}{|c|}{ Impaired GTT } & \multicolumn{2}{|l|}{ Total } \\
\hline & before & after & before & after & before & after \\
\hline \multicolumn{7}{|l|}{$F I L A \mu U / m l$} \\
\hline fasting & $\begin{array}{l}337 \pm 50 \\
(10)\end{array}$ & $399 \pm 146$ & $\begin{array}{l}482 \text { 士 } 148 \\
(15)\end{array}$ & $247 \pm 59$ & $\begin{array}{l}424 \pm 90 \\
(25)\end{array}$ & $308 \pm 66$ \\
\hline $30^{\prime}$ & $\begin{array}{l}516 \pm 132 \\
(10)\end{array}$ & $626 \pm 215$ & $\begin{array}{l}855 \pm 146 \\
(15) x\end{array}$ & $401 \pm 105$ & $\begin{array}{l}719 \pm 100 \\
(25)\end{array}$ & $491 \pm 104$ \\
\hline $60^{\prime}$ & $\begin{array}{l}613 \pm 93 \\
(10)\end{array}$ & $371 \pm 99$ & $\begin{array}{l}1203 \pm 267 \\
(15) x x x\end{array}$ & $566 \pm 124$ & $\begin{array}{l}967 \pm 97 \\
(25) \times x \times x\end{array}$ & $488 \pm 83$ \\
\hline $120^{\prime}$ & $\begin{array}{l}467 \pm 84 \\
(10)\end{array}$ & $436 \pm 133$ & $\begin{array}{l}1182 \pm 258 \\
(15) x\end{array}$ & $484 \pm 135$ & $\begin{array}{l}896 \pm 156 \\
(25) x\end{array}$ & $465 \pm 95$ \\
\hline \multicolumn{7}{|l|}{$I R I \mu U / m l$} \\
\hline fasting & $\begin{array}{l}30 \pm 6 \\
(12)\end{array}$ & $32 \pm 5$ & $\begin{array}{l}41 \pm 6 \\
(15)\end{array}$ & $29 \pm 3$ & $\begin{array}{l}36 \pm 4 \\
(27)\end{array}$ & $30 \pm 3$ \\
\hline $30^{\prime}$ & $\begin{array}{l}77 \pm 10 \\
(12)\end{array}$ & $75 \pm 12$ & $\begin{array}{l}96 \pm 12 \\
(15) x\end{array}$ & $65 \pm 9$ & $\begin{array}{l}88 \pm 8 \\
(27)\end{array}$ & $69_{ \pm}-7$ \\
\hline $60^{\prime}$ & $\begin{array}{l}78 \pm 8 \\
(12)\end{array}$ & $102 \pm 16$ & $\begin{array}{l}132 \pm 15 \\
(15) x\end{array}$ & $92 \pm 10$ & $\begin{array}{l}108 \pm 9 \\
(27)\end{array}$ & $96 \pm 9$ \\
\hline $120^{\prime}$ & $\begin{array}{l}90 \pm 15 \\
(12)\end{array}$ & $101 \pm 19$ & $\begin{array}{l}149 \pm 16 \\
(14) x\end{array}$ & $94 \pm 12$ & $\begin{array}{l}122 \pm 11 \\
(26)\end{array}$ & $97 \pm 10$ \\
\hline $180^{\prime}$ & $\begin{array}{l}66 \pm 14 \\
(10)\end{array}$ & $73 \pm 19$ & $\begin{array}{l}107 \\
(13)\end{array}$ & $71 \pm 11$ & $\begin{array}{l}89 \pm 11 \\
(23)\end{array}$ & $72 \pm 10$ \\
\hline \multicolumn{7}{|l|}{$M I L A \mu U / m L$} \\
\hline fasting & $\begin{array}{l}179 \pm 28 \\
(10)\end{array}$ & $150 \pm 19$ & $\begin{array}{l}157 \pm 26 \\
(13)\end{array}$ & $220 \pm 42$ & $\frac{167}{(23)}$ & $190 \pm 25$ \\
\hline $30^{\prime}$ & $\begin{array}{l}291 \pm 87 \\
(10)\end{array}$ & $258 \pm 49$ & $\begin{array}{l}283 \pm 83 \\
(13)\end{array}$ & $248 \pm 69$ & $\begin{array}{l}286 \pm 58 \\
(23)\end{array}$ & $253 \pm 43$ \\
\hline $60^{\prime}$ & $\begin{array}{l}334 \pm 74 \\
(10)\end{array}$ & $287 \pm 89$ & $\begin{array}{l}343 \pm 88 \\
(13)\end{array}$ & $339 \pm 69$ & $\begin{array}{l}339 \pm 58 \\
(23)\end{array}$ & $316 \pm 54$ \\
\hline $120^{\prime}$ & $\begin{array}{l}317 \pm 86 \\
(10)\end{array}$ & $237 \pm 53$ & $\begin{array}{l}465 \pm 94 \\
(13)\end{array}$ & $320 \pm 64$ & $\begin{array}{l}400 \pm 64 \\
(23)\end{array}$ & $284 \pm 42$ \\
\hline
\end{tabular}

For abbreviations see Table 2 .

For degree of significance see Table 3.

1000 Kalorien Diät. Der vorher stark erniedrigte Quotient FFS/Glycerin normalisierte sich unter der Reduktionsdiät bei beiden Teilkollektiven weitgehend.

b) Cholesterin, Triglyceride, Esterfettsäuren im Nüchternblut. Sowohl der Cholesterin- als auch der Triglycerid-Spiegel fielen (Tabelle 5) während der Behandlung leicht ab, der Unterschied ließ sich jedoch statistisch vicht sichern. Hingegen war der Rückgang

\section{Einfluß auf die Seruminsulinspiegel:}

Bei der Untersuchung der am Fettgewebe bestimmten insulinähnlichen Aktivität (FILA) fand sich die bekannte Erhöhung der Insulinwerte bei Adipösen, vor allem 1, 2 und 3 Std nach Glucoseabgabe im Sinne eines verzögerten und verstärkten Konzentrationsan. stieges. Wie Tabelle 7 und Abb. 2 zeigen, betrifft er jedoch vorwiegend die Gruppe mit abnormer KH- 
Toleranz. Bei dieser Patientengruppe lagen unter der Reduktionsdiät die FLLA-Werte 30,60 und $120 \mathrm{~min}$ nach Glucosegabe signifikant niedriger als vorher, während sich bei dem Teilkollektiv mit normaler Glucosetoleranz keine eindeutigen Veränderungen ergaben. Das ausgeprägte Absinken der Insulinspiegel bei den Patienten mit eingeschränkter KH-Toleranz führte

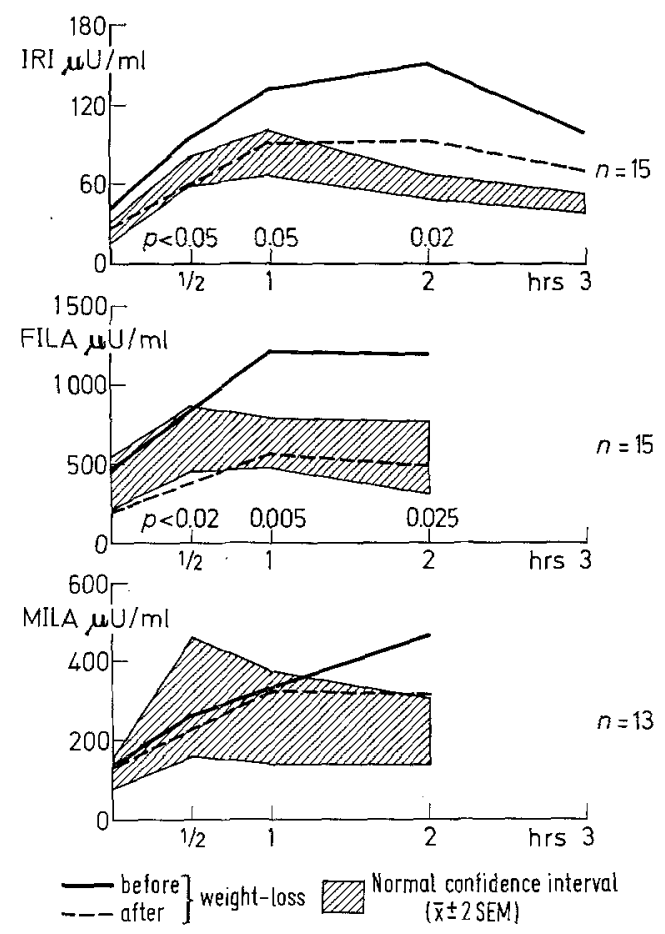

Fig. 2

auch im Gesamtkollektiv zu einem signifikanten Abfall der 60- und 120-Minuten-Werte.

Die Untersuchungen des immunologisch reagierenden Insulins zeigten einen ähnlichen, allerdings etwas weniger stark ausgeprägten Rückgang der Insulinämie, der nur für die Gruppe mit herabgesetzter Glucosetoleranz zu sichern war, während bei der Vergleichsgruppe sogar ein leichter Anstieg eintrat.

Die Werte der am Muskel nachweisbaren insulinähnlichen Aktivität ließen vor und während der Reduktionsperiode keine sicheren Unterschiede erkennen.

\section{Diskussion}

Bereits 1922 hatten PaULIN und SaULS [65] darauf hingewiesen, daß bei Übergewichtigen die Glucosetoleranz häufig beeinträchtigt ist. Diese Befunde sind inzwischen vielfach bestätigt worden $[16,17,24,27$, $43,63,78,79,86]$.

Nach mehrtägigem Fasten sahen die meisten Untersucher bei übergewichtigen Personen mit latentem oder manifestem Diabetes eine Besserung der Kohlenhydrattoleranz $[6,11,39,76,77,78,90]$. So wurde bei 14 adipösen Diabetikern vor und nach Reduktion um 3 bis $13 \mathrm{~kg}$ durch Fasten bei vergleichbarer Diät vor den Kontrolluntersuchungen ein Absinken des Nüch- ternblutzuckers und des Insulinbedarfs beschrieben [39]. Diese Toleranzverbesserung durch Fasten wird. jedoch nicht bei allen Adipösen mit latentem oder manifestem Diabetes beobachtet [5, 31, 77]. Bei normal- und übergewichtigen Personen mit normaler Glucosetoleranz kann Fasten auch zu einer Toleranzver. schlechterung, ,Hunger-Diabetes“" [4, 31, 37], führen.

In Übereinstimmung mit der Mehrzahl der Beobachtungen nach Fastenperioden fanden wir auch bei Gewichtsreduktion durch niederkalorische Kost eine deutliche Toleranzbesserung bei den Adipösen mit gestörter Glucosetoleranz, wobei sich diese in der Hälfte der Fälle sogar völlig normalisierte. Das Teilkollektiv mit normalem Kohlenhydratstoffwechsel zeigte keine eindeutigen Veränderungen.

Von besonderem Interesse ist in diesem Zusammenhang das Verhalten der FFS, die vor allem bei Adipösen mit abnormer KH.Toleranz wesentlich über den Normalwerten Jiegen [19, 33, 42, 57, 61]. Fastenperioden führen zu erheblichen Anstiegen der FFS-Spiegel als Ausdruck vermehrter Lipolyse bei eingeschränkter Rückveresterung [14, 19, 23, 31, 35, 80, 82, 83]. Dieser Anstieg nach Fasten ist bei Adipösen mit und ohne Kohlenhydrattoleranzstörung prozentual geringer ausgeprägt als bei Normalpersonen [35]. Eine Reihe von Autoren $[14,35,45,81,85]$ bezieht die Verschlechterung der Kohlenhydrattoleranz bei Adipösen auf die bei diesem Personenkreis erhöhten FES-Spiegel in Anlehnung an Hales und Randle [70]. Diese und andere Autoren wiesen nach, daß Zugabe von Fettsäuren die Glukoseutilisation am Muskel sowohl in vivo als auch in vitro senkt [30, 64]. GENUTH fand allerdings bei seinen Untersuchungen keinen Zusammenhang zwischen Toleranzverschlechterung and den Spiegeln der FFS und der Beta-Hydroxybuttersäure [31]. Die verbesserte KH-Toleranz der Adipösen mit vorher abnormer GTT nach Reduktionsdiät war bei unseren Fällen nicht von einem Abfall der freien Fettsäuren begleitet, vielmehr kam es zu einem leichten Anstieg, der sich allerdings vorwiegend auf das Teilkollektiv mit normaler Glucosetoleranz beschränkte. Auch war dieser Anstieg insgesamt wesentlich schwächer ausgeprägt als bei den Untersuchungen nach Fastenperioden $[31,32,35,37]$.

Adipöse weisen gegenüber Normgewichtigen erhöhte Nüchternspiegel des freien Serumglycerins auf $[10,92]$, das im Hungerzustand verzögert und wesentlich geringer ansteigt als bei Normalpersonen, so daß eine verzögerte Stimulation der Lipolyse angenommen werden kann [35].

Der von uns unter Reduktionsdiät beachtete erhebliche Abfall des freien Glycerins im Nüchternserum bei gleichzeitigem leichten Anstieg der freien Fettsäuren mit Normalisierung des Quotienten FFS/Glycerin ist damit wahrscheinlich nicht nur als Ausdruck einer partiellen Normalisierung der erhöhten Basallipolyse des Übergewichtigen zu deuten, sondern könnte auch für eine unter diesen Umständen gesteigerte Verwertung des Glycerins in der Peripherie sprechen [23]. 
Die Literaturangaben über das Verhalten des Cholesterinspiegels im Nüchternserum nach Gewichtsreduktion durch Fasten oder niederkalorische bzw. Formuladiät variieren. Mehrfach wurde ein Absinken beschrieben $[29,44,87]$, andere Autoren fanden keine wesentliche Veränderung [12, 58, 59, 68], sogar Anstiege wurden beobachtet [22]. Wir sahen in beiden Teilkollektiven bei nur geringfügig erhöhten Ausgangswerten einen leichten, nicht signifikanten Rückgang.

Triglyceride und Körpergewicht sind schwach positiv korreliert [75]. Bei unseren Untersuchungen lagen vor allem in der Gruppe mit eingeschränkter Kohlenhydrattoleranz die Triglycerid-Spiegel deutlich über der Norm. Nach der Reduktionsdiät sahen wir in beiden Kollektiven einen Rückgang, der allerdings statistisch nicht zu sichern war, während die ebenfalls deutlich über die Normwerte erhöhten Esterfettsäuren beim Teilkollektiv mit normaler KH-Toleranz in den Normbereich zurückkehrten.

Seit langem ist bekannt, daß übergewichtige Personen im Hungerzustand weniger als Normalgewichtige $\mathrm{zu}$ Ketose neigen $[26,46,55]$. Doch wurden auch bei Adipösen schon nach 5-tägigem Fasten Anstiege des Serum-Azetoazetat-Spiegels beobachtet, die die obere Normgrenze um das Zwei- bis Dreifache übersteigen [21]. Der durch diese Ketonämie hervorgerufenen Appetitsenkung wird ein wesentlicher therapeutischer Effekt bei Fastenkuren zugeschrieben (21], wobei die Azetonausscheidung gleichzeitig eine sichere Kontrolle der Diäteinhaltung erlaubt [40].

In unserer Serie kam es nur zu leichten Anstiegen der Beta-Hydroxybuttersäure und des Azetoazetats, so daß der Einfluß der Ketonämie auf die Änderungen der Glucosetoleranz [25] gering zu veranschlagen ist.

Schon 1935 hatte OGILvir bei der Fettsucht einen Hyperinsulinismus vermutet [63]. Diese Annahme ist für Adipöse mit manifestem Diabetes $[1,17,31,45,76$, $86,90]$, mit latentem Diabetes $[48,76,83]$ und evtl. auch solche mit normaler KH-Toleranz $[16,48,66,67$, 76] experimentell bestätigt worden. Welchen Einfluß das Körpergewicht auch bei einem genetisch determinierten Personenkreis auf das Seruminsulin ausübt, zeigen besonders deutlich Untersuchungen an Personen, deren Eltern beide an einem manifesten Diabetes litten [43]. Auch VaGUe [86] fand das immunologisch reagierende Seruminsulin wesentlich stärker vom Körpergewicht als von der genetischen Belastung abhängig, jedoch vor allem mit der bei Adipösen erhöhten 17-OHCS-Ausscheidung korreliert.

Über den Einfluß des Fastens auf das Seruminsulin liegen zahlreiche, zum Teil widersprüchliche Befunde vor. Bei Ratten beobachteten SamaAn u. Mitarb. [76] ein fast völliges Verschwinden der typischen und atypischen ILA nach 7-tägigem Fasten. Beim Menschen wurden an zumeist kleineren Kollektiven Anstiege $[37,76,77,85,90]$, ein leichter Abfall [31] oder keine wesentlichen Änderungen beobachtet [77, 82, 90]. Im Gegensatz dazu liegen über das Verhalten des Seruminsulins unter Reduktionsdiäten nur wenige
Beobachtungen vor. KaRam u. Mitarb. beschrieben bei einem adipösen Diabetiker nach Reduktion von 20 auf $5 \%$ Übergewicht durch 900 Kalorien Diät einen deutlichen Rückgang der Blutzucker- und IRI-Spiegel [45]. RUdNICK und TAYLOR [74] sahen hingegen bei 3 adipösen, frisch entdeckten Diabetikern nach Gewichtsabnahme durch KH-Einschränkung (90 g pro Tag) ein leichtes Ansteigen des IRI.

Bei unseren Untersuchungen Jagen, wie aus Tabelle $7 \mathrm{zu}$ entnehmen ist, bei den Adipösen ohne Glucosetoleranzstörung die nach den angegebenen Methoden bestimmten Spiegel der insulinähnlichen Aktivitäten im jeweiligen Normbereich. Analog zum Verhalten der Blutzuckerspiegel zeigte lediglich das Teilkollektiv mit eingeschränkter KH-Toleranz einen Diäteffekt, der in einer signifikanten Senkung der überhöhten FILA und IRI-Spiegel 30,60 und 120 min nach Glucosegabe bestand und der trotz hoher Streuung bei den FILA-Werten so deutlich ausfiel, daß auch das Gesamtkollektiv einen signifikanten Abfall des 60-und 120-Minuten-Wertes aufwies.

Obwohl die Streuung der mit der in vivo Zwerchfellmethode gewonnenen MILA-Werte etwa im Rahmen der bei der Fettgewebsmethode üblichen Varianz lag, zeigten die MILA-Werte keinen sicheren Unterschied zwischen Adipösen mit und ohne KH-Stoffwechselstörung und auch nicht zwischen den Blutentnahmen vor und während Diätbehandlung. Es ist bekannt, daß die Insulinwirkung am Muskel unter anderem durch freie Fettsäuren [18, 30, 41, 64], Ketokörper [25, 88] und einen kürzlich beschriebenen humoralen Faktor in der Albuminfraktion mit einem Molekulargewicht zwischen 5000 und 10000 verringert [91] und durch Anoxie [60] und Arbeit [20, 62] gesteigert wird. Letztere beiden Faktoren dürften bei der Beurteilung der MILA-Befunde, da die Blutabnahmen im Liegen und unter weitgehend ähnlichen Bedingungen. stattfanden, keine Rolle spielen. Wir fanden auch keine stärkeren Veränderungen der Serumspiegel der erwähnten antagonistischen Metabolite. Soweit die stark streuenden MILA-Werte eine Deutung zulassen, ist daher am ehesten anzunehmen, daß unter der Diättherapie der am Muskel wirksame Insulinanteil weniger zurückging als die insulinähnliche Aktivität am Fettgewebe und in zweiter Linie auch das IRI. Dadurch könnte der vermebrte Abbau von Fettgewebe begünstigt werden. Dafür spricht auch, daß, wie in Tabelle 7 gezeigt, unter der Reduktionsperiode die Glucosebelastung zu geringeren Anstiegen der FILA als der IRI-Werte führte.

Diese Befunde und Deutungsversuche müssen, da bisher nur spärliche und teilweise widersprechende Angaben vorliegen, überprüft werden, zumal das Wesen der insulinähnlichen Aktivität noch strittig ist [28, $53,71]$.

Unsere Befunde zeigen eindeutig auf, daß bei Untersuchungen an Adipösen die KH-Toleranz unbedingt berücksichtigt werden muß. Auch lassen sie den günstigen Einfluß einer Reduktionsperiode auf den 
KH-Stoffwechsel und die dadurch bedingte Insulineinsparung deutlicher hervortreten als die bisher volwiegend nach Fastenperioden durchgeführten Untersuchungen. Bei diesen kam es durch Ketokörper und freie Fettsäuren evtl. zu sekundären Beeinträchtigungen der KH-Toleranz mit Rückwirkung auf die Seruminsulinspiegel. Außerdem sind während längerfristiger Fastenperioden Zwischenfälle beobachtet worden [15].

Unsere Untersuchungen weisen darauf hin, daß der bisher in der Pathogenese der Fettsucht diskutierte Hyperinsulinismus adaptiver Natur ist. Zahlreiche Fragen bleiben jedoch weiterhin offen. So kann aufgrund der bisherigen Arbeiten nicht entschieden werden, wieweit die beobachteten Rückbildungen von Stoffwechselveränderungen bei Adipösen durch den latenten Hungerzustand oder aber durch die erzielte Gewichtsreduktion bewirkt wurden, da im allgemeinen die Untersuchungen sofort im Anschluß an die Behandlung stattfanden. Durch weitere Untersuchungen hoffen wir, in diese Fragen besseren Einblick zu erhalten. Erste Befunde sprechen dafür, daß in erster Linie das Ausmaß der Gewichtsabnahme entscheidend ist.

\section{Literatur}

1. Abramson, E., and R.A. Arky: Treatment of the obese diabetic. A comparative study of placebo, sulfonylurea and phenformin. Metabolism 16, 204-212 (1967).

2. ADlersberg, D.: Obesity, fat metabolism and diabetes. Diabetes 7, 236-243 (1958).

3. AlBrink, M.J., and J.W. MaIGs: Interrelationship between skinfold thickness, serum lipids and blood sugar in normal men. Amer. J. clin. Nutr. 15, 255261 (1964).

4. Arnould, Y., E. Balasse, J.R.M. Franckson, and P.A. BAsTínIE : Effect of protracted starvation on the glycoregulation of obese patients. Diabetologia 1, 136 (1965) abstr.

5. Beck, P., J.M.T. Kaumans, C.A. Winterlin, M.F. Stein, W.H. DaUghaday, and D.M. KrPnis : Studies of insulin and growth hormone secretion in human obesity. J. Lab. clin. Med. 64, 654-667 (1964).

6. BERKowirz, D.: Metabolic changes associated with obesity before and after weight reduction. J.A.M.A. 187, 399-403 (1964).

7. BloEM, W.L., G. Azar, J. Clark, and H.J. MAC KAY: Comparison of metabolic changes in fasting obese and lean patients. Ann. N.Y. Acad. Sci. 131, 623-631 (1965).

8. BöнLE, E.: Vortrag anläßlich des Symposiums über Stoffwechselfragen bei der Fettsucht, Düsseldorf 1964.

9. BommermanN, P., P. Schwarz u. K. KopErz: Über das Verhalten der insulinähnlichen Aktivität im Serum bei Fettsucht. Dtsch. med. Wschr. 90, 917-921 (1965).

10. -- R. Schulze-Sölde u. M. DammbacheqR: Untersuchungen über den Fettstoffwechsel bei der Fettsucht. Diabetologia 1, 180-186 (1966).

11. Bouchardat, A.: De la glycosurie au diabète sucré, zit. n. 74. Paris: Germer-Baillière 1875.

12. Caldwell, A.B., P. Watson, D.B. Green, A. FloRIN, P. Braun, and M.L. Bimenenbaum: Weight reduction and serum cholesterol levels. Amer. J. clin. Nutr. 12, 401-405 (1963).

13. Conard, V., J.R.M. Franckson, P.A. Bastente, J. Kasters et L. Kovacs: Etude critique du triangle d'hyperglycémie intraveineux chez l'homme normal et détermination d'un ,,coéfficient d'assimilation glucidique $^{6}$. Arch. int. Pharmacodyn. 93, 277-292 $(1953)$.

14. Corvilain, J.A. Champenais, H. Loeb, and $M$. ABRAMOW: Effect of fasting on levels of plasma-nonesterified fatty acids in normal children, normal adults and obese adults. Lancet 1961 I, 534-535.

15. Cubberlex, P.T., S.S.A. Polster, and C.I. SohulMAN : Lactic acidosis and death after the treatment of obesity by fasting. New Engl. J. Med. 272, 628$630(1965)$.

16. Daweke, H., H. van Landeghem, I. Bach, H. ZimMERMANN u. A. BreITBACH: Bestimmung der insulinähnlichen Aktivität und der physiologischen Insulinreserve bei schwerer Adipositas. Klin. Wschr. 43, $185-190(1965)$.

17. - - W. Winkelmann u. I. Bach: Der Einfluß der Adipositas auf die insulinähnliche Aktivität und physiologische Insulinreserve beim Altersdiabetes. Klin. Wschr. 43, 190-196 (1965).

18. Delorme, M.: Hemmung der Insulinwirkung durch die freien Fettsäuren beim Hund. Klin. Wschr. 43, $729-732$ (1965).

19. DoLE, V.P.: A relation between nonesterified fatty acids in plasma and the metabolism of glucose. J. clin. Invest. 35, 150-154 (1956).

20. DUHN, W.E., and J.J. ClarK: Studies concerning a possible humoral factor produced by working muscles. Its influence on glucose utilization. Diabetes 10, 289 297 (1961).

21. Duncan, G.G., J.K. Jenson, F.C. Cristofori, and G.L. SCHLESS: Intermittent fasts in the correction and control of intractable obesity. Amer. J. med. Sci. 245, $515-520(1963)$.

22. ENDE, N.: Starvation studies with special reference to cholesterol. Amer. J. clin. Nutr. 11, 270-280 (1962).

23. Ermer, K.P., K. Schwarz u. K.F. Whinges: Über das Verhalten der unveresterten Fettsäuren und des Glycerins im Hungerzustand nach Glukose und Fruktose-Gaben. Verh. dtsch. Ges. inn. Med. 67, 699-702 (1961).

24. Felber, J.P., N. Zaragoza, L. Grasste, A.J. Moody et A. VANNOTTI: Utilisation diminuée du glucose et accumulation de graisse chez lobèse diabétique. Schweiz. med. Wschr. 96, 1575-1578 (1966).

25. Felts, P.W., O.B. Crofford, and C.R. Park: Effect of infused ketone bodies on glucose utilization in the dog. J. clin. Invest. 43, 638-646 (1964).

26. FoutIN, O., and W. Dernis: On starvation and obe. sity. J. biol. Chem. 21, 183-192 (1915).

27. Fratorson, J.R.M., W. Matarse, Y. Arnould, E. Rasto, H. A. Ooms, E. Balasse, V. Conard, and P.A. BAstenre: Glucose kinetics in human obesity. Diabetologia 2, 96-103 (1966).

28. FroEsch, E. R. : Minkowski-lecture. VI. Congr. Intern. Diab. Feder. 30. VII bis 4. VIII 1967, Stockholm.

29. Galibraith, W.B., W.E. Conror, and D.B. Stone: Weightloss and serum lipid changes in obese subjects given low calorie diets of varied cholesterol content. Ann. int. Med. 64, 268-275 (1966).

30. Gartand, P.B., E.A. Newshomme, and P.J. RaNdLe: Regulation of glucose uptake by muscle. Part. 9. Biochem. J. 93, 665-678 (1964).

31. GENUTH, S.M.: Effects of prolonged fasting on insulin secretion. Diabetes 15, 798 - 806 (1966).

32. Gordon, E.S.: Nonesterified fatty acids in the blood of obese and lean subjects. Amer. J. clin. Nutr. 8, $740-745(1960)$.

33. - Relationship between obesity and diabetes mellitus. Metabolism 11, 819-832 (1962). 
34. Grady, H.J., and M.A. Lamar: Glucose determination by automatic chemical analysis. Clin. Chem. 5, $542-550(1959)$.

35. Gries, F.A., G. Zimmer u. K. JAHNKe: Fettstoffwechselstudien bei Adipositas. Verh. dtsch. Ges. inn. Med. 70, 424-427 (1964).

36. Hales, C.N., and P.J. Randle: Immunoassay of insulin with insulin-antibody precipitate. Biochem. J. $88,137-146(1963)$

37. - - Effect of low carbohydrate diet and diabetes mellitus on plasma concentrations of non-esterified fatty acids, glucose and insulin during oral glucosetolerance tests. Lancet 1963 I, $790-794$.

38. Hartmann, E., R. Schmid, and M. Sotarrattar: Metabolic studies on obese patients during starvation. Helv. med. Acta 33, $66-75$ (1966).

39. Harrison, M.T., and R.M. Harden: The long-term value of fasting in the treatment of obesity. Lancet 1966 II, $1340-1342$.

40. Heyden-Stucky, S.: Gewichtszunahme, Übergewicht, Gewichtsreduktion. Schweiz. med. Wschr. 97, $78-81(1967)$

41. Hirche, H., u. S. Kolke: Hemmung der Aufnahme und des Abbaues der Glukose im Herzmuskel narkotisierter Hunde durch die freien Fettsäuren. Pflügers Arch. ges. Physiol. 280, 158-164 (1964).

42. JaHnke, K., F.A. Gries, H. WaLlenfels u. $H$ Schulte: Verhalten von Metaboliten des Fettstoff wechsels im Serum adipöser und nicht adipöser Personen unter Grundumsatzbedingungen. Klin. Wschr. 42, 1016-1020 (1964).

43. - H. Daweke, W.H. Schilling, R. RÜenauver u. K. Oberdisse: Der potentielle Diabetes (sog. Praediabetes). Verh. dtsch. Ges. inn. Med. 72, 851-868 (1967).

44. Jolliffe, N., S.H. Rinzler, and M. Archer: Prudent reducing diet: effect on serum cholesterol, study of 111 obese men 50-59 years old. Arch. intern. Med. $109,566-572(1962)$.

45. Karam, J.H., G.M. Grodsky, and P.H. Forsham: The relationship of obesity and growth hormone to serum insulin levels. Ann. N.Y. Acad. Sci. 131, 374387 (1965)

46. Kexwick, A., G.L.S. Pawan, and T.M. Chatmers: Resistance to ketosis in obese subjects. Lancet 1959 II, $1157-1159$.

47. Kleine Nährwerttabelle der Dtsch. Ges. f. Ernährung e.V. Hrsgb. W. WIRTHs Frankfurt/Main: Umschau Verlag, 13. Auflage, 1965.

48. Kreisberg, R.A., B.R. Bosheli, J. Diplacido, and R. F. RoDDAM : Insulin secretion in obesity. New Engl. J. Med. 276, 314-319 (1967).

49. KREUTz, F.H.: Enzymatische Glycerinbestimmung. Klin. Wschr. 40, 362-363 (1962).

50. - Enzymatic determination of glycerol in the measurement of triglycerides. Int. Congr. Clin. Chem. Detroit (1963).

51. Liebermeister, H., H. G. Solbach, W.H. Schtluing, H. Meissner, D. GrüneKLeE u. H. DawkKe: Vergleichende Untersuchungen der insulinähnlichen Aktivitäten am Muskel, am Fettgewebe und mit einer radioimmunologischen Methode bei Akromegalie. (In Vorbereitung)

52. LochNer, W., u. M. NASSERI: Untersuchungen über den Herzstoffwechsel und die coronare Durchblutung insbesondere bei Dinitrophenolvergiftung. Pflügers Arch. ges. Physiol. 271, 405-419 (1960).

53. Lyngsøe, J.: Seruminsulin. A review. Acta. med. scand. Suppl. to vol. 179.

54. Martin, D.B., A.E. Renold, and Y.M. Dagerais: An assay for insulinlike activity using rat adipose tis. sue. Lancet 1963 II, $76-77$.
55. Mc KaY, E.M., and J.W. SherRTL: A comparison of the ketosis developed during fasting by obese patients and normal subjects. Endocrinology 21, 677 -680 (1937).

56. Meduey, D. R.K.: The relationship between diabetes and obesity: A study of susceptibility to diabetes in obese people. Quart. J. Med. 34, 111-132 (1965).

57. Mennert, H., L. Pelikan, u. N. Zöllner: Über die Konzentration der freien Fettsäuren im Serum von Diabetikern und Fettsüchtigen. Klin. Wschr. 39, 888 -891 (1961).

58. Moore, N.S., C.M. Young, and L.A. Maynard: Blood lipid levels as influenced by weight reduction in women. Amer. J. Med. 17, $348-354$ (1954).

59. - J.H. Fryer, C.M. Young, and L.A. Maynard: Blood lipid levels as influenced by weight reduction in men. Amer. J. clin. Nutr. 3, $397-402$ (1955).

60. Morgan, H. E., E. Cadenas, D.M. Regen, and C.R. PARK: Regulation of glucose uptake in muscle. II. Rate-limiting steps and effects of insulin and anoxia in heart muscle from diabetic rats. J. biol. Chem. 236, $262-268$ (1961).

61. Morse, W.I., and R. Mahabir: Changes in glucose tolerance and plasma free fatty acids after fasting in obesity. Diabetes 13, 286-290 (1964).

62. NeELy, J.R., H. Liebermeister, and H.E. Morgan: Fffect of pressure development on membrane trans port of glucose in isolated rat heart. Amer. J. Physiol. 212, 815-822 (1967).

63. OgrLvie, R.: Sugar tolerance in obese subjects: A review of 65 cases. Quart. J. Med. 28, 345-358 (1935).

64. Parmeggiani, A., and R.H. Bowman: Regulation of phosphofructokinase activity by citrate in normal and diabetic muscle. Biochem. Biophys. Res. Comm. 12, 268-273 (1963)

65. Paullin, J.E., and H.C. Sautus: A study of the glucose tolerance test in the obese. Sth. med. J. (Bgham, Ala.) 15, 249-253 (1922).

66. PERley, M., and D.M. KtPNIS: Plasma insulin responses to glucose and tolbutamide of normal weight and obese diabetic and nondiabetic subjects. Diabetes 15, 867-877 (1966).

67. Phenr, D.N.: The normal and diabetic patterns of insulin response to glucose. Lancet 1962 II, $955-958$.

68. Potndexter, C.A., and M. Brugers: Effect of low calorie diets and resultant weight loss on plasma cholesterol in the obese. Arch. intern. Med. 56, 884890 (1935).

69. RAFAELSEN, O.: Insulin like activity of human serum determined by glycogen increase of diaphragm after intraperitoneal injection. Acta physiol scand. 61, 323 $-330(1964)$

70. Randle, P.J., P. B. Garland, C.N. Hales, and E.A. Newsholme: The glucose-fatty acid cycle. Its role in insulin sensitivity and the metabolic disturbances of diabetes mellitus. Lancet 1963 I, 785-789.

71. Rasio, E.A., J.S. Soemdner, and G.F. Cahrul: Insulin and insulin-like activity in serum and extracellular fluid. Diabetologia 1, 125-127 (1965).

72. Remadte, B.: Vergleichende Untersuchungen mit der oralen und der i.v. Glukosebelastung unter gleichzeitiger Berücksichtigung des i.v. Tolbutamidtestes bei fettleibigen Personen. Diss. Düsseldorf (1967) im Druck.

73. Rosenthal, H.L., M.L. Pfutue, and I. Calierame The colorimetric estimation of serum fatty acids. Clin. chim. acta 4, 329-333 (1959).

74. RUDNICK, P.A., and K.W. TAYLOR: Effect of prolonged carbohydrate restriction on serum insulin levels in mild diabetes. Brit. med. J. 1965 I, 1225-1228.

75. SaILER, S., F. SANdHOFER, and H. BraUnsteiner: Overweight and Triglyceride level in normal persons 
and patients with diabetes mellitus. Metabolism 15, $135-137(1966)$.

76. Samaan, N., J. Brown, R. Fraser, and I. Trayner: Effect of obesity and of starvation on insulin activity. Brit. med. J. 1965 I, 1153-1156.

77. SCHALCH, D.S.: Changes in carbohydrate tolerance in obese diabeties during starvation: Relationship to alterations in the secretion of insulin, glucagon and growth hormone. Diabetes 15, 527 (1966) abstr.

78. SCHLESS, G.L., and G.G. DuncaN: The beneficial effect of intermittent total fasts on the glucose tolerance in obese diabetic patients: Metabolism 15, 98-102 (1966).

79. Schilling, W.H., K. Oberdisse, K.A. Hüter u. H. BLANK: Vergleichende Untersuchungen mit der oralen und intravenösen Glucosebelastung zur Erfassung einer verminderten Kohlenhydrattoleranz. Diabetologia 1, 187-194 (1966).

80. Schwarz, E., H.G. vaN RIET, and W. Schapman: Serum growth hormone and energy supply in fasting obese patients. Metabolism 15, 294-305 (1966).

81. Schrade, W., E. Böhle, R. Btegler u. H. Kirst: Untersuchungen über die unveresterten Fettsäuren des Blutes. Münch. med. Wschr. 101, 2062-2067 (1959).

82. Sowldner, J.S., J. Stminkw, M.G. HmrRera, and G.F. CAHmL: Effect of seven-day-fast on glucosetolerance and serum insulin of six normal males. Endocrin. Society June 17 (1965) p. 66. abstr.

83. Sussmann, K.E.: Effect of prolonged fasting on glucose and insulin metabolism in exogenous obesity. Arch. intern. Med. 117, 343-347 (1966).

84. Technicon Arbeitsvorschrift nach: Zlatkis A., B. ZAK, and A.J. BoYle: A new method for the direct determination of serum cholesterol. J. Lab. clin. Med. 41, $486-492$ (1953).
85. Unger, R.H., A.M. Eisentratt, and L. L. Mapison : The effects of total starvation upon the levels of circulating glucagon and insulin in man. J. clin. Invest. 42, 1031- 1039 (1963).

86. Vague, P., R. Depieds, G. Boeuf, J.L. Codacciont et J. VAGUE: Quelques facteurs de l'hyperinsulinisme de certaines obésités. Diabetologia 2, 224 (1966) abstr.

87. WALKER, W.J., LoWRY E.Y., D.E. Love, G.V. Mann, S.A. Levine, and F.J. Stare: Effect of weight reduction and caloric balance on serum lipoprotein and cholesterol levels. Amer. J. Med. 14, 654-664 (1953).

88. Wriciamson, J.R., and H.A. Krebs: Acetoacetate as fuel of respiration in the perfused rat heart. Biochem. J. 80, 540-549 (1961).

89. - J. Mellanby, and H.A. KreBs: Enzymic determination of $\mathrm{D}(-) \cdot \beta$ 'hydroxybutyric acid and acetoacetic acid in blood. Biochem. J. 82, 90-96 (1962).

90. YaLOW, R.S., S.M. Glick, J. Roth, and S.A. Berson: Plasma insulin and growth hormone levels in obesity and diabetes. Ann. N.Y. Acad. Sci. 131, 357-373 $(1965)$.

91. Young, D.A.B.: A serum inhibitor of insulin action on muscle. I. Its detection and properties. Diabetologia 3, 287-298 (1967).

92. Zimmer, G., F.A. Gries, u. K. Jahnke: Das Verhalten von Metaboliten des Fettstoffwechsels im Serum adipöser und nichtadipöser Personon bei oraler Kohlenhydratzufuhr und kurzfristiger Nahrungskarenz. Klin. Wschr. 42, 1020-1024 (1964).

Dr. H. LTEBERMEISTER

II. Medizinische Klinik und

Poliklinik der Universität

4000 Düsseldorf, Moorenstr. 5 